\title{
Early Guillain-Barré syndrome in coronavirus disease 2019 (COVID-19): a case report from an Italian COVID-hospital
}

\section{Donatella Ottaviani ${ }^{1}$ • Federica Boso ${ }^{1,2}$ - Enzo Tranquillini ${ }^{1} \cdot$ Ilaria Gapeni $^{1} \cdot$ Giovanni Pedrotti $^{3}$ - Susanna Cozzio ${ }^{4}$. Giovanni M Guarrera ${ }^{5} \cdot$ Bruno Giometto $^{1}$ (10)}

Received: 10 April 2020 / Accepted: 2 May 2020 / Published online: 12 May 2020

(C) Fondazione Società Italiana di Neurologia 2020

\begin{abstract}
Guillain-Barré syndrome (GBS) is an acute polyradiculoneuropathy associated with dysimmune processes, often related to a previous infectious exposure. During Italian severe acute respiratory syndrome coronavirus-2 outbreak, a woman presented with a rapidly progressive flaccid paralysis with unilateral facial neuropathy after a few days of mild respiratory symptoms. Coronavirus was detected by nasopharyngeal swab, but there was no evidence of its presence in her cerebrospinal fluid, which confirmed the typical albumin-cytological dissociation of GBS, along with consistent neurophysiological data. Despite immunoglobulin infusions and intensive supportive care, her clinical picture worsened simultaneously both from the respiratory and neurological point of view, as if reflecting different aspects of the same systemic inflammatory response. Similar early complications have already been observed in patients with para-infectious GBS related to Zika virus, but pathological mechanisms have yet to be established.
\end{abstract}

Keywords Coronavirus disease 2019 (COVID-19) · SARS-CoV-2 neurological complications · Guillain-Barré syndrome . Para-infectious Guillain-Barré syndrome

Guillain-Barré syndrome (GBS) is an acute, immunemediated polyradiculoneuropathy with a wide range of clinical manifestation; neuromuscular respiratory failure occurs in up to $30 \%$ of patients, leading to mechanical ventilation and intensive care unit admission. $60 \%$ cases are estimated to be related to an infection, most frequently with gastrointestinal or respiratory symptoms. Besides the demonstration of

Ottaviani Donatella and Boso Federica contributed equally to this work.

Bruno Giometto

bruno.giometto@apss.tn.it

1 Neurology Unit, Rovereto Hospital, Azienda Provinciale per i Servizi Sanitari (APSS) di Trento, Trento, Italy

2 Department of Neuroscience, Biomedicine and Movement Sciences, University of Verona, Verona, Italy

3 Intensive Care Unit, Rovereto Hospital, Azienda Provinciale per i Servizi Sanitari (APSS) di Trento, Trento, Italy

4 Internal Medicine Unit, Rovereto Hospital, Azienda Provinciale per i Servizi Sanitari (APSS) di Trento, Trento, Italy

5 Health Trust, Azienda Provinciale per i Servizi Sanitari (APSS) di Trento, Trento, Italy molecular mimicry between epitopes of $C$. jejuni and nerves, many microorganisms have been investigated in its pathogenesis, e.g., the recent reports of Zika virus (ZIKV)-related GBS [1]. During the current pandemic, one adult case of GBS has been associated with Coronavirus disease 2019 (COVID-19) [2], but it is not clear if this strain of coronavirus (severe acute respiratory syndrome coronavirus-2, SARS-CoV-2) might also have a potential for neurological complications rather than be coincidentally present in neurological patients.

We report the case of a 66-year-old woman, coming from a COVID-19 endemic area, who presented with a 72-h history of increasing difficulty walking and acute fatigue; she had mild fever and cough 10 days earlier, with spontaneous resolution of fever after a few days. She also manifested a transient pruriginous dorsal rash but had no gastrointestinal symptoms nor relevant medical history, besides mild hypertension treated with beta-blockers. Lung CT scan showed bilateral groundglass opacities, but the first rhinopharyngeal swab for SARS$\mathrm{CoV}-2$ was negative; initial routine and gas analyses on blood were unremarkable (Table 1). On evaluation, she was paraparetic with a rapidly progressive symmetric weakness in the lower limbs, leading to falls and paraplegia. She also had initial distal weakness in the upper limbs (MRC 4/5) 
Table 1 Blood routine analyses and arterial blood gas tests of our patient, at admission and on the day of the acute clinical worsening that led to intensive care unit (ICU)

\begin{tabular}{llll}
\hline & On admission & ICU admission & Unit of measurement and normal range \\
\hline StO2 $(\%)$ & $95.1 \%$ & $75 \%$ & \\
pO2 & 72.3 & 48.9 & $\operatorname{mmHg}(75.0-100)$ \\
pCO2 & 26.4 & 33.6 & $\operatorname{mmHg}(35.0-45.0)$ \\
pO2/FiO2 & 344 & 106 & $\mathrm{mmHg}(350-480)$ \\
$\mathrm{Hb}$ & 12.3 & 9.7 & $\mathrm{~g} / \mathrm{dL}(12-16)$ \\
WBC & $8.3 * 10^{9}$ & $4.5 * 10^{9}$ & $\mathrm{Cells} / \mathrm{L}\left(4-10^{*} 10^{9}\right)$ \\
Lymphocytes & $0.9 * 10^{9}$ & $/$ & $\mathrm{Cells} / \mathrm{L}\left(1.4 * 10^{9}\right)$ \\
Platelets & $500^{*} 10^{9}$ & $129 * 10^{9}$ & $\mathrm{~L}(150-400)$ \\
D-dimer & 326 & 506 & $\mathrm{ng} / \mathrm{mL}(<150)$ \\
CK & 456 & 124 & $\mathrm{U} / \mathrm{L}(26-192)$ \\
CRP & 7.8 & 70.6 & $\mathrm{mg} / \mathrm{L}(6)$ \\
Creatinine & 0.8 & 0.9 & $\mathrm{mg} / \mathrm{L}(0.5-1)$ \\
GGT & 61 & 182 & $\mathrm{U} / \mathrm{L}(5-50)$ \\
AST & 37 & 48 & $\mathrm{U} / \mathrm{L}(5-40)$ \\
ALT & 37 & 56 & $\mathrm{U} / \mathrm{L}(10-65)$ \\
Albumin & 38 & 24 & $\mathrm{~g} / \mathrm{dL}(35-50)$ \\
INR & 1.01 & 1.13 & $(0.85-1.15)$ \\
LAD & 395 & 183 & $\mathrm{U} / \mathrm{L}(87-241)$ \\
\hline
\end{tabular}

StO2 oxygen saturation, $\mathrm{pO} 2$ partial pressure of oxygen, $\mathrm{pCO} 2$ partial pressure of carbon dioxide, $\mathrm{FiO} 2$ fraction of inspired oxygen, $H b$ hemoglobin; $W B C$ white blood cells, $C K$ creatinine kinase, $C R P$ C-reactive protein, $G G T=$ gamma-glutamyl transpeptidase, $A S T$ aspartate transaminase, $A L T$ alanine transaminase, INR international normalized ratio, $\mathrm{LAD}=$ lactate dehydrogenase and diffuse areflexia, but no clear sensory deficits. She underwent lumbar puncture with demonstration of a typical albumin-cytological dissociation (0 cells/uL, $108 \mathrm{mg} / \mathrm{dL}$ proteins), thus prompting initiation of intravenous immunoglobulin (IVIg, $0,4 \mathrm{~g} / \mathrm{kg}$ for 5 days) on the presumptive diagnosis of GBS. Ten days after onset, a nerve conduction study disclosed absence of F-waves with diffuse prolonged distal motor latencies and reduced distal compound muscle action potential amplitudes with a slight reduction of conduction velocities, thus suggesting a mixed pattern of demyelination and axonal damage; no sensory nerve action potential was registered (Table 2). SARS-CoV-2 was not detected on cerebrospinal fluid (CSF), while serology was not available at that time; anti-glycolipid antibodies were also absent.

In the meanwhile, the patient had been admitted to a subintensive care unit as her second swab resulted positive for SARS-CoV-2 and thus started combined treatment of antiretroviral drugs (lopinavir and ritonavir) and hydroxychloroquine, maintaining reasonable respiratory function with supplemental oxygen.

However, the woman did not benefit from early treatment with IVIg and progressively developed proximal weakness in all limbs, dysesthesia, and unilateral facial palsy. She also needed treatment for transient episodes of confusion with psychomotor agitation. Moreover, gas exchanges worsened with a sudden desaturation, requiring intubation and intensive care unit admission, where she was treated for multi-organ failure along with a leg deep vein thrombosis and a superimposed bacterial infection ( $a b$ ingestis pneumonia).

\section{Discussion}

The clinical picture of our patient was in keeping with GBS according to Brighton criteria, with increasing flaccid weakness of the limbs, areflexia, albuminocytologic dissociation, and consistent neurophysiologic data. Other causes of concurrent neurotoxicities were also excluded, as hydroxichloroquine generally accounts for different side effects and antiretrovirals had yet to be administered when she started having neurological symptoms. Intensive supportive care and the need for strict isolation impaired clinical and instrumental assessment of the neurological aspect, despite being critical to determine prognosis. 
Table 2 Preliminary nerve conduction studies (NCS), which were performed 10 days after presentation to the emergency department for lower limbs weakness, when the patient was isolated in the COVID-intensive care unit

\begin{tabular}{|c|c|c|c|c|c|c|}
\hline Motor NCS & Stimulation point & Record point & Distal latency (ms) & Amplitude (mV) & Velocity (m/s) & $\begin{array}{l}\text { F wave minimal } \\
\text { latency }(\mathrm{ms})\end{array}$ \\
\hline \multirow[t]{2}{*}{ Left tibial nerve } & Ankle & \multirow[t]{2}{*}{ Abductor hallucis brevis } & 13.9 & 3.5 & & \multirow[t]{2}{*}{ / } \\
\hline & Popliteal fossa & & 26.7 & 3.0 & 33.6 & \\
\hline \multirow[t]{2}{*}{ Left common peroneal nerve } & Ankle & \multirow[t]{2}{*}{ Extensor digitorum brevis } & 13.6 & 2.3 & & \multirow[t]{2}{*}{ I } \\
\hline & Head of fibula & & 22.5 & 1.9 & 38.7 & \\
\hline \multirow[t]{2}{*}{ Right median nerve } & Wrist & \multirow[t]{2}{*}{ Abductor pollicis brevis } & 6.6 & 3.1 & & \multirow[t]{2}{*}{ l } \\
\hline & Elbow & & 11.0 & 2.6 & 47.7 & \\
\hline \multicolumn{2}{|l|}{ Antidromic sensory NCS } & Record point & Stimulation point & Latency (ms) & \multicolumn{2}{|l|}{ Amplitude $(\mu \mathrm{V})$} \\
\hline \multicolumn{2}{|l|}{ Right median nerve } & Wrist & 3rd digit & 1 & \multicolumn{2}{|l|}{1} \\
\hline \multicolumn{2}{|l|}{ Right ulnar nerve } & Wrist & 5th digit & / & \multicolumn{2}{|l|}{ / } \\
\hline \multicolumn{2}{|l|}{ Right radial nerve } & Wrist & 1st digit & / & \multicolumn{2}{|l|}{ / } \\
\hline \multicolumn{2}{|l|}{ Right sural nerve } & Lateral malleolus & Calf & / & \multicolumn{2}{|l|}{ / } \\
\hline
\end{tabular}

Remarkably, contrary to the previous reported case with COVID-19 [2], our patient developed neurological deficits a week after the onset of respiratory symptoms and they progressed simultaneously; therefore, the evolution of her clinical picture does not support the typical post-infectious pattern of GBS and it could rather resemble a form of acute para-infectious paralysis that has already been associated with some viruses, such as ZIKV [3]. Indeed, this ZIK-V manifestation has peculiar characteristics that led to speculation about a possible different pathogenesis compared to classical GBS: (a) they seem to arise 5 to 10 days after infection and have higher morbidity, more frequent cranial neuropathies and concurrent immune-mediated disorders such as thrombocytopenic purpura [4]; (b) anti-glycolipids antibodies typically involved in cross-reactive mechanisms are not common [5]; (c) there is a predominant involvement of distal nerve segments [3], where the blood-nerve barrier is lacking.

The mechanisms underlying a para-infectious pathogenesis have yet to be established, but in some predisposed patients, ZIKV might generate an aberrant immune response that, in turn, causes GBS; in particular, it has been proposed that specific ZIKV-peptides might not only cross-react but also induce a cellular immune reaction through antigen-presenting cell activation of T-lymphocytes [6].

Likewise, the timing of neurological signs in our case may be too rapid to imply a heterologous immune reaction to a first viral exposure and it might suggest other pathogenic mechanisms, such as direct viral damage (i.e., CMV and VZV neuritis) or acute dysimmune processes that arise as a part of systemic inflammatory response (which has been presumed on the basis of lung involvement in itself). The growing number of extrapulmonary manifestations of COVID-19, the immunological nature of GBS itself, the absence of SARS-CoV2 in the CSF and the delayed onset of paralysis in our patient might theoretically support the second hypothesis rather than a viral effect.

Specific serological assays were not available at the time of her examination, but the hypothesis of neurological manifestations of COVID-19 is supported by the evidence of neurotropic and neuroinvasive characteristics of coronaviruses in both mice and humans [7]; moreover, other strains of human coronaviruses have already been involved with peripheral nervous system manifestations [8,9] and with pediatric cases of GBS $[10,11]$.

Along with dedicated research, it will be crucial to monitor the incidence of GBS in the coming months, as a concurrent increase in cases during COVID-19 outbreak would further support a possible causal relation. ${ }^{1}$

\section{Compliance with ethical standards}

Ethical approval The patient consented to publish her anonymized health data.

Conflict of interest The authors declare that they have no conflict of interest.

\footnotetext{
${ }^{1}$ While the article was under review, 5 similar cases of GBS in patients with COVID-19 were reported by another Italian group [12].
} 


\section{References}

1. Cao-Lormeau VM, Blake A, Mons S, Lastère S, Roche C, Vanhomwegen J, Ghawché F (2016) Guillain-Barré syndrome outbreak associated with Zika virus infection in French Polynesia: a case-control study. Lancet 387(10027):1531-1539

2. Zhao H, Shen D, Zhou H, Liu J, Chen S (2020 [published online ahead of print, 2020 Apr 1]) Guillain-Barré syndrome associated with SARS-CoV-2 infection: Causality or coincidence? Lancet Neurol S1474-4422(20):30109-30105

3. Uncini A, Shahrizaila N, Kuwabara S (2017) Zika virus infection and Guillain-Barré syndrome: a review focused on clinical and electrophysiological subtypes. J Neurol Neurosurg Psychiatry 88: 266-271

4. Musso D, Ko A, Baud D (2019) Zika virus infection - after the pandemic. N Engl J Med 381:1444-1457

5. Shang P, Zhu M, Wang Y et al (2020) Axonal variants of GuillainBarré syndrome: an update. J Neurol [published online ahead of print, 2020 Mar 5]

6. Muñoz LS, Parra B, Pardo CA (2017) Neurological implications of Zika virus infection in adults. J Infect Dis 216(Suppl 10):S897S905

7. Desforges M, Le Coupanec A, Brison É, Meessen-Pinard M, Talbot PJ (2014) Neuroinvasive and neurotropic human respiratory coronaviruses: potential neurovirulent agents in humans. In: Adhikari R, Thapa S (eds) Infectious Diseases and Nanomedicine I. Advances in Experimental Medicine and Biology, vol 807

8. Tsai L, Hsieh S, Chao C et al (2004) Neuromuscular disorders in severe acute respiratory syndrome. Arch Neurol 61(11):1669-1673

9. Kim JE, Heo JH, Kim HO, Song SH, Park SS, Park TH, Ahn JY, Kim MK, Choi JP (2017) Neurological complications during treatment of middle east respiratory syndrome. J Clin Neurol 13(3):227233

10. Sharma K, Tengsupakul S, Sanchez O, Phaltas R, Maertens P (2019) Guillain-Barre syndrome with unilateral peripheral facial and bulbar palsy in a child: A case report. SAGE Open Med Case Rep 7:2050313X19838750

11. Turgay C, Emine T, Ozlem K, Muhammet SP, Haydar AT (2015) A rare cause of acute flaccid paralysis: human coronaviruses. J Pediatr Neurosci 10:280-281

12. Toscano G, Palmerini F, Ravaglia S, Ruiz L, Invernizzi P, Cuzzoni MG, Franciotta D, Baldanti F, Daturi R, Postorino P, Cavallini A, Micieli G (2020) Guillain-Barré syndrome associated with SARSCoV-2. N Engl J Med [published online ahead of print, 2020 Apr 17]

Publisher's note Springer Nature remains neutral with regard to jurisdictional claims in published maps and institutional affiliations. 